\title{
Start or End? ; One of the Biggest Mysteries is Finally Solved?
}

\section{Keiji Ueda*}

Division of Virology, Department of Microbiology and Immunology, Osaka University Graduate School of Medicine, Japan

\begin{abstract}
Hepatitis B virus (HBV) is a small DNA virus, which was found a half century ago. No useful and convenient in vitro infection system for HBV has hampered detailed analysis of HBV life cycle, which leads to development of HBV related diseases. Here, Yan et al. reported this November, 2012 that a Sodium Taurocholate Cotansporting Polypeptide called also NTCP was a receptor for HBV and established an HBV in vitro infection system using ordinary hepatocellular carcinoma derived cell lines. This finding will shed light on total elucidation of the HBV life cycle and lead to development of new anti-HBV drugs.
\end{abstract}

It is now a half century since hepatitis B virus (HBV) was identified by Blumberg et al. [1]. This virus is a small DNA virus whose genome is partially double stranded circular DNA and is only $3.2 \mathrm{~kb}$ in size. The viral genes are compactly organized and four genes; core, $\mathrm{S}$, polymerase and $\mathrm{X}$ are identified [2]

There are about 360 million people infected with the virus worldwide, forming one of the biggest infectious diseases and one of the most serious health programs of human being [3]. HBV infection causes not only severe acute hepatitis including fulminant hepatitis that is highly lethal, but also chronic hepatitis leading liver cirrhosis and hepatocellular carcinoma especially through vertical infection. Treatment against HBV infection consists of interferon therapy and anti-viral drugs. The interferon therapy is, however, very limited and not as effective as that against Hepatitis C Virus (HCV) infection [4]. And furthermore, anti-HBV drugs, all of which are nucleoside analogues, were developed as anti-Human Immunodeficiency Virus (HIV) drugs not as anti-HBV ones and the HBV polymerase specificity and peculiarity were not considered. Needless to say, both viruses have a reverse transcription process of their replication cycles and thus reverse transcriptase activity work in a similar way, i.e., their activity center is composed of an MYDD motif [2]. These kinds of anti-viral drugs always lead to generation and expansion of mutants that escape their effectiveness.

As mentioned, exploitation and development of new really effective drugs against HBV considering its viral life cycle and gene functions is one of the most important issues for human health. But we have not had very useful HBV infection system in vitro and in vivo to study detail viral life cycle and its pathogenesis. Hepadnaviruses are highly species specific and tissue specific and HBV never infects present hepatocyte-originated human hepatocellular carcinoma cell lines such as HepG2. Although there are several animal hepadnaviruses; avians (Duck Hepatitis B Virus [DHBV] and Heron Hepatitis B Virus [HHV]), rodents (Woodchuck Hepatitis Virus [WHV] and Ground Squirrel Hepatitis Virus [GSHV]) and primates for HBV [5,6], it is not easy to hatch and raise these kinds of animal and to prepare primary hepatocytes from these animals.

As for in vitro infection systems, two systems are available at present; one is Primary Human Hepatocytes (PHH) or tree shrew (Tupaia belangen) [7] which is also susceptible to HBV as human and chimpanzee and another is HepaRG [8], a cultured Human Hepatocellular Carcinoma (HCC) cell line established from $\mathrm{HCV}$ caused HCC.

These in vitro systems facilitate our knowledge about how HBV infects hepatocytes, but still not good enough because of their commercialism, invariability dependent on the lot and takes cost for preparation and time to induce infectivity.

A recent splendid report by Yan et al. may improve such inconvenience to study HBV [8]. For long time, we have been searching for HBV receptors and several molecules including those from the DHBV system were nominated as HBV receptors but they had never contributed establishment for HBV infection systems in vitro, though many data have suggested that the ligand on the HBV side for the receptor must be in the preS1 region [9-11]. This time, Yan et al. [8] successfully pulled down an HBV receptor, a sodium taurocholate cotansporting polypeptide called also NTCP or NTCP-1, with photoactivating preS1 peptide using PTH. I would say that many researchers had been trying to elucidate HBV receptors with the same kind methods including phage libraries screening with the preS1 region; synthetic peptides or the protein purified from $E$. coli, insect cells and yeast expression/purification system etc., and wonder why such a molecule has not been picked up until now.

Yan et al. showed that NTCP expression was low in common HCC cell lines but high in $\mathrm{PHH}$ and $\mathrm{PTH}$, and that introduction of NTCP expression raised the competency of $\mathrm{HBV}$ in these unsusceptible cell lines and knockdown of NTCP in PTH lowered the susceptibility. Thus, a tip of the mystery has been finally solved by Yan et al.? Though this must be re-evaluated by the other researchers, the finding will shed light on total elucidation of the HBV life cycle and lead to development of new anti-HBV drugs.

\section{References}

1. Blumberg BS, Alter HJ, Visnich S (1965) A "New" Antigen in Leukemia Sera. JAMA 191: 541-546.

2. Seeger C, Zoulim F, Mason WS (2007) Hepadnaviruses. Lippincott Williams and Wilkins, Philadelphia.

3. Shepard CW, Simard EP, Finelli L, Fiore AE, Bell BP (2006) Hepatitis B virus infection: epidemiology and vaccination. Epidemiol Rev 28: 112-125.

*Corresponding author: Keiji Ueda, Division of Virology, Department of Microbiology and Immunology, Osaka University Graduate School of Medicine, 2-2 Yamada-oka, Suita, Osaka 565-0871, Japan E-mail: kueda@virus.med.osaka-u.ac.jp

Received December 19, 2012; Accepted February 28, 2013; Published March 04, 2013

Citation: Ueda K (2013) Start or End? ; One of the Biggest Mysteries is Finally Solved? J Med Microb Diagn 2: 121. doi:10.4172/2161-0703.1000121

Copyright: (c) 2013 Ueda K. This is an open-access article distributed under the terms of the Creative Commons Attribution License, which permits unrestricted use, distribution, and reproduction in any medium, provided the original author and source are credited. 
4. Zoulim F (2006) Antiviral therapy of chronic hepatitis B. Antiviral Res 71: 206215

5. Menne S, Cote PJ (2007) The woodchuck as an animal model for pathogenesis and therapy of chronic hepatitis B virus infection. World J Gastroenterol 13: 104124.

6. Funk A, Mhamdi M, Will H, Sirma H (2007) Avian hepatitis B viruses: molecular and cellular biology, phylogenesis, and host tropism. World J Gastroenterol 13: 91-103.

7. Lanford RE, Chavez D, Brasky KM, Burns RB, Rico-Hesse R (1998) Isolation of a hepadnavirus from the woolly monkey, a New World primate. Proc Natl Acad Sci U S A 95: 5757-5761.

8. Gripon P, Rumin S, Urban S, Le Seyec J, Glaise D, et al. (2002) Infection of a human hepatoma cell line by hepatitis B virus. Proc Natl Acad Sci U S A 99: $15655-15660$.

9. Yan H, Zhong G, Xu G, He W, Jing Z, et al. (2012) Sodium taurocholate cotransporting polypeptide is a functional receptor for human hepatitis $\mathrm{B}$ and $\mathrm{D}$ virus. eLife 1: e00049.

10. Neurath AR, Kent SB, Strick N, Parker K (1986) Identification and chemical synthesis of a host cell receptor binding site on hepatitis $B$ virus. Cell 46: 429436 .

11. Engelke M, Mills K, Seitz S, Simon P, Gripon P, et al. (2006) Characterization of a hepatitis $B$ and hepatitis delta virus receptor binding site. Hepatology 43 750-760.

12. Glebe D, Urban S (2007) Viral and cellular determinants involved in hepadnavira entry. World J Gastroenterol 13: 22-38. 